\title{
Advanced Diagnostic Aids in Early Detection of Oral Cancer
}

Pankhuri Nigam $^{1} \&$ Vijay Pal Singh²

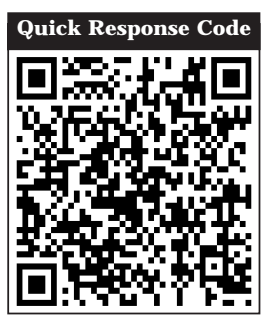

doi: $10.5866 / 2014.631596$

${ }^{1 \& 2}$ Post Graduate student

Dept of Oral Pathology,

Shree Bankey Bihari Dental College,

Ghaziabad, U.P. India

\section{Article Info:}

Received: April 13, 2014

Review Completed: May 13, 2014

Accepted: J une 12, 2014

Available Online: October, 2014 (www.nacd.in)

(c) NAD, 2014 - All rights reserved

Email for correspondence:

pankhurinigam85@gmail.com

\begin{abstract}
:
With the increase in prevalence of oral cancer seen worldwide, early screening and advanced diagnostic techniques are playing an important role in early diagnosis and treatment planning of patients. The five year survival rate of oral cancer remains low for the past three decades and delayed diagnosis has been suggested to be one of the major reasons. Commonly used method of diagnosis indudes histopathology and visualization adjuncts such as toulidene blue, vizilite etc. The present article reviews the research on development of new and upcoming tools for early and accurate diagnosis of oral cancer.
\end{abstract}

Key words: advances, oral cancer, diagnostic techniques

\section{NTRODUCTION}

Approximately $94 \%$ of all oral malignancies are squamous cell carcinoma (SCC). The annual incidenceand mortality rates vary between different races, genders, and age groups. I n the U nited States this is 7.7 per $100,000 .^{1}$

Persons with oral SCC almost have been aware of an alteration in that site for 4-8 months before seeking professional help. There is minimal pain during the early growth phase and this may explain the delay in seeking professional care. If the health care professional does not have a high index of suspicion, additional several weeks or months may elapse before a biopsy is performed. ${ }^{1}$
Accounting for $96 \%$ of all oral cancers, squamous cell carcinoma (SCC) is usually preceded by dysplasia presenting as white epithelial lesions on the oral mucosa. Leukoplakias develop in $1-4 \%$ of the population. Malignant transformation, which is quite unpredictable, develops in $1-40 \%$ of leukoplakias over 5 years. Dysplastic lesions in the form of erythroplakias carry a risk for malignant conversion of $90 \% .^{2}$ Tumor detection is further complicated by a tendency towards field cancerisation, leading to multicentric lesions. ${ }^{3}$

Screening and an early detection are believed to decrease both the morbidity and mortality which are associated with oral SCC, because unlike many 
anatomic sites, in the oral cavity, pre-malignant lesions are often visible on clinical examination. However, an accurate discrimination between premalignant vs reactive/inflammatory lesions via conventional visual and tactile examinations alone is problematic. As the malignant potential of oral lesions cannot be accurately predicted solely on the basis of their clinical characteristics, a histological evaluation is essential for all suspicious lesions. The definition of an oral mucosal pre-malignancy that is based on a conventional histologic examination can also be problematic. Lesions are currently considered as pre-cancerous when there are cytomorphologic changes which are consistent with dysplasia. However, the various criteria for diagnosing and grading dysplasia are controversial, highly subjective and open to wide range of interpretation, even among highly qualified pathologists. In addition, no definitive criteria currently exist for predicting the risk of a cancerous transformation of individual dysplasticlesions; even dysplastic oral lesions have been reported to undergo spontaneous regression.

Therefore, conventional histologic findings can only be utilized to indicate that a given lesion may have a malignant potential, and that it cannot be used for the prediction of a malignant change. Hence, two key issues should be considered:

1. In general, a progression to oral SCC many not occur in a linear fashion over a uniform period of time. Rather, thereare subsets of lesions with histologic evidences of dysplasia, that may or may not progress to oral SCC.

2. Similarly, the histologically normal appearing mucosal lesions may truly be benign or they may represent molecular premalignant lesions that have not yet developed morphologic/cytologic changes which are consistent with dysplasia. ${ }^{4}$

Routinely used diagnostic aids includetoulidene blue, Oral brush biopsy, Vizilite, cytology etc.

\section{DISADVANTAGES OF ROUTINE DIAGNOSTIC AIDS}

1. Toulidene Blue - Low positive predictive value of $43.5 \%$ for potentially malignant lesions and a false negative rate as high as $20.5 \%$ for premalignant lesions also have been reported. ${ }^{5}$

2. Brush Cytology (Oral CDx) - Many studies have reported specificity as low , around 32\%; and a high false positive rate was reported as ranging from 43 to $89 \%{ }^{5}$

3. Adjunct based on tissue reflectance (ViziLite, ViziLite Plus, Microlux) - Several studies have investigated the benefit of using the ViziLite compared to conventional visual examination alone. Most of these studies either did not include comparison to the diagnostic gold standard i.e histopathological diagnosis based on scalpel biopsy, or contained only a limited number of cases subsequently biopsied which made it difficult to assess specificity and sensitivity. There was only one study that correlated the ViziLite findings to the histopathologic diagnosis and the sensitivity, specificity and accuracy were reported to be $100 \%, 0 \%$ and $18.2 \%$ respectively. ${ }^{5}$

\section{EMERGING DIAGNOSTIC ADJ UNCTS}

LAB ON A CHIP - Microfuidics technologyalso referred to as lab-on-a-chip or micro-totalanalysis systems (TAS)- is the adaptation, miniaturization, integration and automation of analytical laboratory procedures into a single device or "chip". Microfluidics is often regarded as the chemistry or biotechnology equivalent of the silicon integrated silicon chip that has revolutionized electronics, computers and communications. ${ }^{6}$

Microfluidics are by definition suited for handling living cells (whose typical diameter is a few micrometers) in a three-dimensional, biologically rel evant environment. ${ }^{7}$ This microfluidic chip accepts saliva sample, can be operated by minimally trained personnel, and can provide a diagnostic answer in an automated and timely fashion. The detection of oral pre-cancer (dysplastic) and cancer cells within the chip will take advantage of membrane-associated cell proteins that are singularly expressed on cell cancer cells. The measured profile is compared with archived gene transcription profiles to determine the cancer type 
and stage. As such, this system provides a means for automated, rapid detection and molecular analysis of cancers in a miniaturized format suitable for use in the clinic and/or the operating room. ${ }^{7}$

\section{OPTICAL COHERENCE TOMOGRAPHY}

Optical coherence tomography (OCT) was first reported by Fujimoto et al. in 1991. OCT has been widely used in numerous clinical applications, including gastroenterology, ophthalmology, dermatology, and dentistry. OCT is a non-invasive, non-radiative optical diagnostic tool based on interferometers. By using a low-coherence broadband near-infrared light source, it is possible to obtain excellent spatial resolution ( $\sim 20$ ì $\mathrm{m}$ ) and real-time images.

Optical coherence tomography (OCT) has been proved to be a useful technique for oral disease diagnosis. In particular, based on the scanning images of a swept-source OCT system, a few effective diagnosis indicators for oral cancer and precancer have been identified. These indicators include the EP layer thickness and the standard deviation (SD) of OCT signal intensity. In an abnormal oral EP containing dysplastic cells, the cell size, shape, nucleus size, and arrangement become more randomly distributed, when compared with healthy oral epithelium (EP). In this situation, light scattering becomes stronger and its spatial distribution becomes more strongly fluctuated. The light scattering behaviors are recorded in the spatial variations of OCT signal intensity. It is noted that the spatially average scattering intensity in a dysplastic oral EP is generally higher than a normal oral EP such that the corresponding average OCT signal intensity is relatively higher. Therefore, although the overall signal intensity may vary from one OCT image to another, the SD value without the normalization with respect to the average can better describe the rough morphology in a dysplastic oral EP. A normalization procedure will significantly blur the feature of stronger OCT intensity fluctuations in space. Statistics based on clinical practice has shown the high sensitivity and specificity in using those diagnosis indicators. The
SD calculation has also been used for diagnosing submucous fibrosis. In such a diagnosis, the decrease of the SD level in the LP layer due to collagen deposition is observed. ${ }^{9}$

The advantage of OCT includes cross-sectional images of normal or abnormal tissues can be obtained without biopsy and no preparation of the sample or patient is required. As it is an optical technique and uses the light for imaging the tissues there is no exposure of the patient to ionizing radiation. ${ }^{10}$

\section{FLUORESCENCE ENDOSCOPY}

Fluorescence diagnostic imaging is a relatively new technique that involves the use of a photosensitising drug to visualize lesions through tissue fluorescence. Results from studies using 5aminolevulinic acid (5-ALA)-induced protophorphyrin IX (PplX) fluorescence have shown this technique to be superior to whitelight endoscopy for the diagnosis of various types of cancer, including those in the oral cavity. Hypericin is a plant-based photosensitiser that accumulates selectively in abnormal cells, including tumour cells. Red fluorescence was observed in lesions, indicating a selective uptake of hypericin in lesions compared with normal tissue. Moreover, hyperplastic and SCC lesions show different levels of fluorescence. Highly vascular tissue is likely to be close to the tissue surface, resulting in brighter fluorescence compared with normal tissue. As hypericin fluoresces in the orange - red region (emission peak about $590 \mathrm{~nm}$ ), the green fluorescence emitted is most likely because of autofluorescence of the tissue. The green-to-blue (G/B) intensity ratio, which can be thought of as autofluorescence normalised to the blue excitation light, showed no particular trend between normal, hyperplastic and SCC tissue (results not shown here). Thus, the G/B ratio may not be a good image parameter for distinguishing tissue types. On the other hand, the red-to-green(R/G) intensity ratio, which can be thought of as a ratio of tissue fluorescence to autofluorescence, could distinguish between normal and hyperplastic tissue and between normal and SCC tissue reasonably well. ${ }^{11}$ 


\section{DIFFUSE REFLECTANCE IMAGING}

Optical spectroscopy provides useful diagnostic information about the morphological and biochemical changes related to progression of cancer in the oral cavity. ${ }^{12}$

When light interacts with biological tissue a small portion of it is absorbed or transmitted while the rest undergoes multiple elastic scattering due to heterogeneity in the refractive index of the tissue components and gets diffusely reflected. Often, a portion of the impinging radiation is reflected from the surface when the roughness of the boundary is small in comparison to wavelength of light. The portion that penetrates the sample gets scattered at a larger number of points in its path due to uneven, broken or bumpy boundary surfaces where the coarseness is of the same order of magnitude as the wavelength. During tissue transformation healthy tissue undergoes morphometric and cytologic changes such as increase in epithelial thickness, nuclear size, nuclear to cytoplasmic ratio, changes in the chromatin texture and collagen content, and angiogenesis. These changes modify the diffusely reflected component of the incoming radiation. ${ }^{13}$

Diffuse Reflectance (DR) is the result of single and multiple backscattering of the white excitation light a property that depends on tissue morphology such as nuclear size, distribution, epithelial thickness, and collagen content, and the amount of oxy and deoxy - haemogl obin presence in the exposed tissue, all of which can vary during tissue transformation to malignant state. Strong proof-ofprinciple for utilization of diffuse reflectance spectroscopy(DRS) , a non-invasive optical tool for early detection of malignant changes in the oral cavity, has emerged recently. ${ }^{12}$

\section{CONCLUSION}

The field of oral pathology and medicine are undergoing changes and we have a comea long way. Advances in diagnosis at the molecular level are expected to affect choice of treatment and patient outcomes. There all practitioners should be aware of the latest advancements and should try to incorporate them whenever feasible.

\section{ACKNOWLEDGEMENT}

I would like to acknowledge Dr. Krishnadeoprasad, H.O.D, Dept of Oral Pathology, S.B.B.D.C, Ghaziabad; Dr. Vineet Gupta, Reader, Dept of Oral Pathology, S.B.B.D.C, Ghaziabad; Dr. J alaj Tak, Reader, Dept. of Oral Pathology, S.B.B.D.C, Ghaziabad; Dr. Anju Sinha, Senior Lecturer, Dept. of Oral Pathology, S.B.B.D.C, Ghaziabad and Dr. Ruchita Bali, Senior Lecturer, Dept. of Oral Pathology, S.B.B.D.C, Ghaziabad.

\section{REFERENCES}

1. Falaki Farnaz, Delavarian Zahra, Pakfetrat Atessa, Mohtasham Nooshin, Shirazian Shiva. Oral Squamous Cell Carcinoma with an unusual clinical manifestation: a case report. Cases J ournal 2009; 2:660-668.

2. Wilder Smith Petra, J ung Woong Gyu, Brenner Matthew, Osann Kathryn, Beydoun Hamza, Messadi Diana et al. In vivo optical coherence tomography for the diagnosis of oral malignancy. Lasers Surg Med 2004; 35:269-275.

3. Slaughter DP. Field cancerization in oral stratified squamous epithelium. Cancer 1953; 6:963-968.

4. Seethalakshmi C. Early Detection of Oral Squamous Cell Carcinoma(OSCC)- Role of Genetics: A Literature Review. J Clin Diag Res 2013; 7(8):1824-1826.

5. Cheng L. Yi-Shing, Wright J ohn. Advances in Diagnostic Adjuncts for Oral Squamous Cell Carcinoma. The Open Pathology J ournal 2011; 5:3-7.

6. Mehrotra and Gupta: Exciting new advances in oral cancer diagnosis: avenues to early detection. Head Neck Onc 2011; 3:33.

7. Virupakshappa Banu. Applications of Nanomedicine in Oral Cancer. OHDM 2012; 1(2):62-68.

8. Hsieh Yao-Sheng, Ho Ching-Yi, Lee Shyh-Yuan, Chuang Ching-Cheng, Tsai J ui-che, Lin Kun-Feng et al. Sensors 2013; 13: 8928-8949.

9. Lee Cheng-Kuang, Chi Ting-Ta, Wu Chiung-Ting, Tsai Meng-Tsan, Chiang Chun- Pin, Yang Chih-Chung. Diagnosis of oral precancer with optical coherence tomography. Biomedical Optic Express 2012; 3(7):16321646.

10. Nagalaxmi V, Prameela K, Zardi FT. Optical Coherence Tomography for Oral Mucosal Lesions - A Review Article. J Adv Med Dent Scie 2013; 1(2):48-52.

11. Thong PSP, Olivo M, Chin WWL, Bhuvaneshwari R, Mancer K, Soo KC. British J ournal of Cancer 2009; 101:1580-1584.

12. J ayanthi J L, Nisha GU, Manju S, et al. Diffuse reflectance spectroscopy: diagnostic accuracy of a non-invasive screening technique for early detection of malignant changes in the oral cavity. BMJ Open 2011; 1:71. 